\title{
CONCEPT OF AN ADAPTIVE-TUNED PARTICLES IMPACT DAMPER
}

\author{
Mateusz Żurawski, Robert Zalewski, Bogumit Chiliński \\ Warsaw University of Technology, Institute of Machine Design Fundamentals, Warsaw, Poland \\ e-mail: mateusz.zurawski@pw.edu.pl; robert.zalewski@pw.edu.pl; bogumil.chilinski@pw.edu.pl
}

\begin{abstract}
The most popular devices for attenuation of mechanical vibrations are dampers or shock absorbers. Two main energy dissipation strategies can be generally distinguished: passive and active (semi-active). Passive vibration isolation methods are the most commonly used, mainly because of their simplicity and low maintenance costs. Among passive vibration attenuation techniques, also Particle Impact Dampers (PID) are involved. Classical PID solutions have some certain limitations. This paper aims at presenting the new concept of an adaptive tuned PID damper that can pretend to be placed among semi-active energy dissipation methods.
\end{abstract}

Keywords: particles impact damper, adaptive-passive damping, smart materials, granular materials

\section{Introduction}

Nowadays, "smart" materials have a significant impact on the engineering industries. Their biggest advantage over classical structures is the possibility of changing physical properties according to the external factor. Typical examples of smart structures are: piezoelectrics, shape memory alloys, rheological fluids (MRF and ERF) or Vacuum Packed Particles (Bartkowski et al., 2019). Due to the dangerous resonance effect, intelligent materials are often involved in the semi-active damping of mechanical vibration processes. Many of popular energy attenuation devices are composed of various types of fluids or advanced structural materials that dissipate energy basing on elongation/compression or shearing modes. Another group of devices incorporate friction phenomena as a main damping factor. In these cases, temperature and wear of materials play an important role in damping properties. To avoid the previously mentioned problems, the particle damping method seems to be especially effective. Particle Impact Damping (PID) strategies have been successfully implemented in civil and vehicles engineering, various machinery fields and aerospace. Although the PID technology is rapidly developing, it is still a form of passive additional mass type damping technique which, in some sense, exhibits some disadvantage over semi-active damping strategies. Usually, particle damping reveals temperature independent behavior. Since 1937, when Paget proposed the PID technique as a competitive to other popular vibration attenuation methods, granular dampers are still improved (Paget, 1937).

For example, a paper by Du (2017) describes the advantages of the PID damper equipped with additional springs. A mathematical model of such an object was proposed and an experimental tests were carried out for the model verification. The results showed that the application of additional damping elements could improve damping capabilities of the investigated system leading to reduction of the vibration amplitude up to $80 \%$ in the resonant range. Various approaches to the mathematical modeling proceses of PID devices can be found in the world literature. They mainly focus on representing the damping effect in the particular system undergoing unwanted vibrations (Snoun and Trigui, 2018; Lei etal, 2018). Despite the simplicity of the principle of a device based on the loose particles, it can be concluded that the phenomena occurring in it are rather complex. Other approaches to PID problems compare the extraordinary 
granular flow inside the investigated system to the flow typical for the gas state. Such a solution allows one to describe the damping phenomenon as a function depending on: mass, volume, restitution coefficient and excitation frequency (Zhang etal, 2016a; Du and Wang, 2010). PID types and their variations are generally used in long, slender, and relatively light objects. Wind turbines are typical examples where granular damping seems to be particularly efficient (Zhang et al., 2016b; Chen and Georgakis, 2013), which is discussed in detail in (Fitzgerald et al., 2018; Hussan et al., 2018). The previously mentioned papers confirmed the possibilities of using PID in such elements to reduce vibrations in unexpected ranges. In this paper, the authors present an innovative solution that employs a Particle Impact Damper to change the dynamic properties of the vibrating cantilever beam in an adaptive-passive way. It is possible thanks to the special design of the damping device enabling rapid changes in the volume of the container filled with a granular material.

\section{Idea of the adaptive tuned PID}

Basing on the previously mentioned papers, it can be stated that the damping properties of PIDs are strongly dependent on the volume of the container, where the loose particles can interact. This feature influences the filling ratio and the character of collisions between single grains and the casing. In this paper, the authors propose an innovative prototype of PID, where the volume of the device can be rapidly changed thanks to the pressure controlled adaptive chamber (Zalewski and Żurawski, 2019). A detailed scheme of the discussed damper is depicted in Fig. 1.

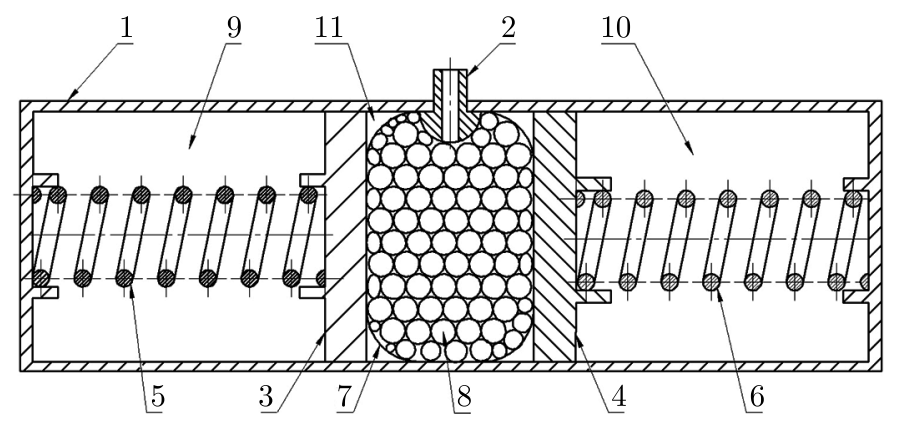

Fig. 1. Scheme of ATPID

The proposed Adaptive Passive Particle Impact Damper (ATPID) is an adjustable vibration attenuator enabling the user to change dynamic properties of vibrating elements. The proposed device consists of rigid casing (1), air valve (2), two movable diaphragams (3), (4), two springs (5), (6), expansible balloon (7), loose particles (8). The ATPID can be divided into three separate subsections (9), (10), (11). In the middle section (9), the rubber balloon (7) filled with loose grains (8) is located. The central section of the device is limited by two movable membranes (3), (4) connected by appropriate springs (5), (6) attached to the outer walls of the casing. The proposed solution allows the user to control the expansion of the rubber element by pumping it in and out. The possibility of changing the volume of the rubber element by connecting it to the external pump results in dynamical and fully controlled changes of the volume of the compartment where the particles collisions take place. Such a mechanism allows for introducing an innovative semi-active damping of vibrations strategy. To confirm the effectiveness of the proposed damping methodology, a special test stand was designed and developed. It consisted of a cantilever beam and an additional ATPID element that could be fixed along its entire length. 


\section{The model}

For the preliminary approach, a 2-DOF mathematical model was assumed (1). In Fig. 2, a mechanical scheme of the model is presented. $M$ denotes the reduced mass of the beam and the ATPID element. The values of stiffness $K$ and damping $C$ parameters of the considered beam

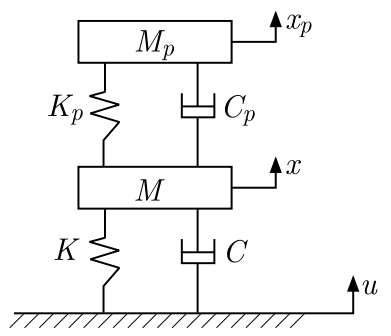

Fig. 2. Mathematical model

were defined basing on the initial experimental results. $M_{p}$ stands for the mass of the grains material. The value of the $K_{p}$ parameter was captured experimentally for the turned off ATPID element and was assumed to be constant during experiments

$$
\begin{aligned}
& {\left[\begin{array}{cc}
M & 0 \\
0 & M_{p}
\end{array}\right]\left[\begin{array}{c}
\ddot{X} \\
\ddot{X}_{p}
\end{array}\right]+\left[\begin{array}{cc}
K+K_{p} & -K_{p} \\
-K_{p} & K_{p}
\end{array}\right]\left[\begin{array}{c}
X \\
X_{p}
\end{array}\right]+\left[\begin{array}{cc}
C+C_{p} & -C_{p} \\
-C_{p} & C_{p}
\end{array}\right]\left[\begin{array}{c}
\dot{X} \\
\dot{X}_{p}
\end{array}\right]} \\
& +\left[\begin{array}{cc}
-C & 0 \\
0 & 0
\end{array}\right]\left[\begin{array}{c}
\dot{u} \\
0
\end{array}\right]+\left[\begin{array}{cc}
-K & 0 \\
0 & 0
\end{array}\right]\left[\begin{array}{l}
u \\
0
\end{array}\right]=\left[\begin{array}{l}
0 \\
0
\end{array}\right]
\end{aligned}
$$

Additional damping $C_{p}$, resulting from inelastic collisions of the grains can be described as

$$
C_{p}=C_{A T P I D}\left(L, h, \dot{x}_{p}, f, \alpha, \rho_{p}, d, e\right) A(t)+C_{p_{0}}
$$

where $C_{A T P I D}$ is a damping coefficient dependent on the volume of the container and particles ratio $\alpha, C_{p_{0}}$ is an initial damping of the system for the compacted grains (ATPID is turned off), $e$ - the restitution coefficient, $L$ and $h$-geometrical parameters, $x_{p}$ - velocity of particles, $f$ - excitation frequency, $\rho_{p}$ - density of particles, $d$ - diameter of grains. $A(t)$ is activation function (3.3) depicted in Fig. 3

$$
\begin{gathered}
A(t)=\frac{a\left(t-t_{\text {start }}\right)}{t_{1}}\left[H\left(t-t_{\text {start }}\right)-H\left(t-t_{2}\right)\right]+a H\left(t-t_{2}\right) \\
-\frac{a\left(t-t_{\text {stop }}\right)}{t_{3}}\left[H\left(t-t_{\text {stop }}\right)-H\left(t-t_{4}\right)\right]-a H\left(t-t_{4}\right)
\end{gathered}
$$

where: $a$-amplitude, $t$-time, $t_{1}$ - activation time, $t_{2}$ - operating time, $t_{3}$ - deactivation time, $t_{4}$ - time of the turned off state, $t_{\text {start }}$ - turning on time, $t_{\text {stop }}$ - turning off time, $H$ - Heaviside function.

In this paper, the authors do not want to focus on the detailed description of the calibration process of the $C_{A T P I D}$ parameter value. The problem is quite complex and demands carrying out additional laboratory tests including various volumes of the grains container. The value of the considered parameter was determined experimentally only for the "on" and "off" states of the damper separately (compacted and fully released state). In this preliminary approach, the additional assumption was made that the $C_{A T P I D}$ paremeters variations are a linear function of the container volume. Such an approach is an obvious simplification and will be subjected to further investigations. The main objective of this paper is just to reveal the innovative adaptive passive controlling properties of the investigated ATPID system. 


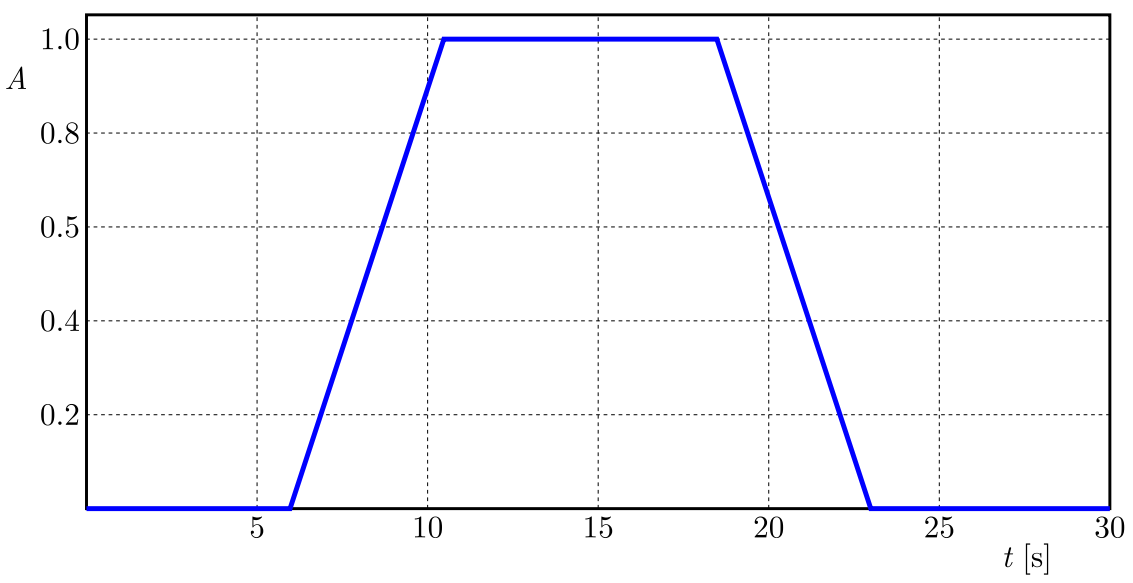

Fig. 3. Activation function

(a)

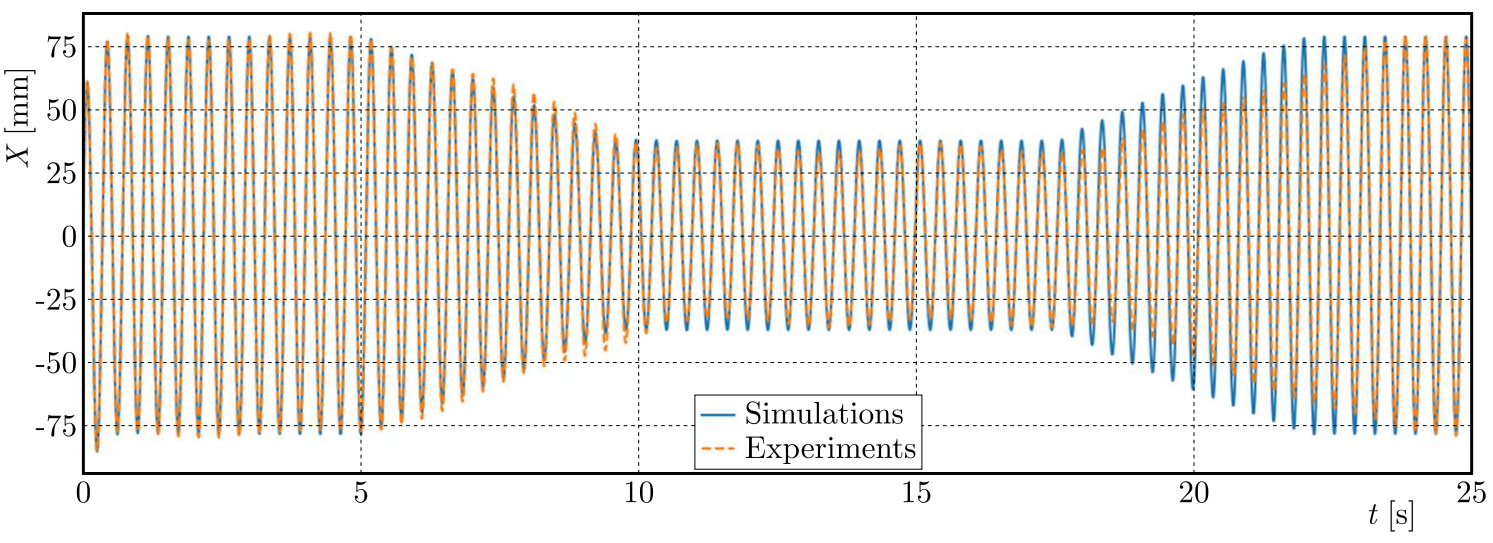

(b)

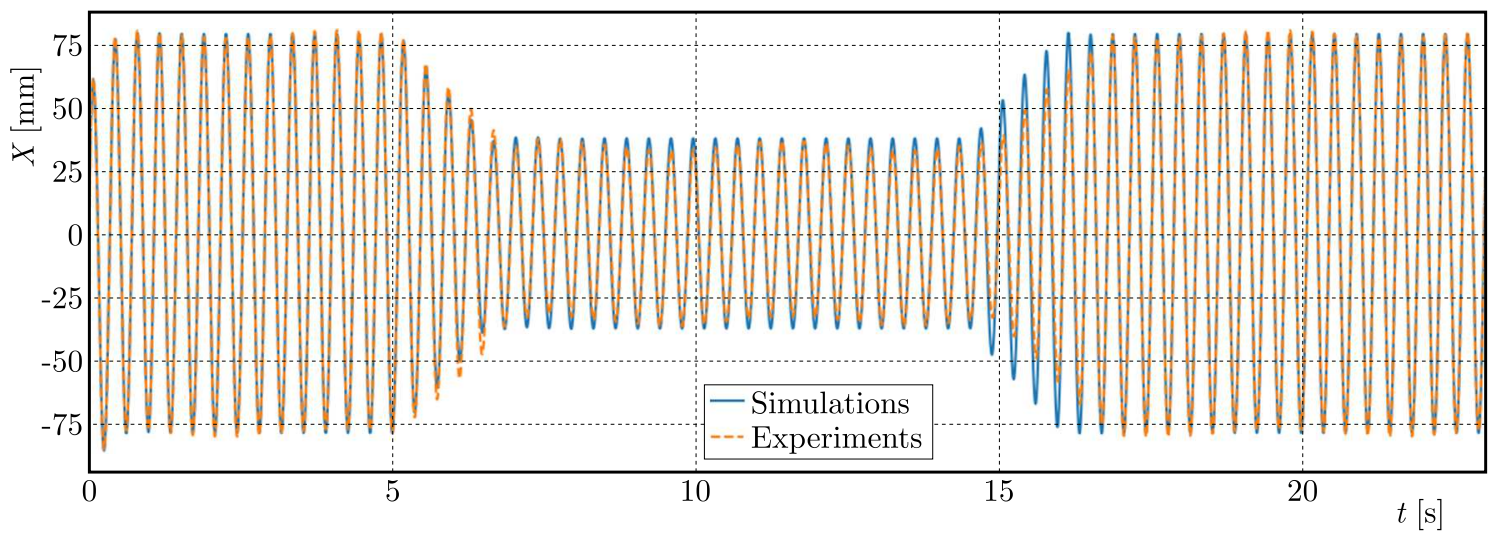

Fig. 4. Comparison of experiments and simulation; (a) $t_{1}=4.5 \mathrm{~s}$, (b) $t_{2}=1.5 \mathrm{~s}$

\section{Experiments and simulations}

The most important stage of the research was the analysis of beam vibrations in the resonance range during the switching "on" and "off" of the ATPID. Two different activation functions were taken into consideration in this paper. They correspond to the fully inflating time of the balloon in $4.5 \mathrm{~s}$ and $1.5 \mathrm{~s}$, respectively.

The experimentaly investigated cantilever beam with a rectangular cros-section (width $35 \mathrm{~mm}$, high $3 \mathrm{~mm}$ ) and length $500 \mathrm{~mm}$ was subjected to a kinematic excitation. The vibrations of the investigated element were measured by an accelerometer. For the considered case, the applied resonant excitation frequency was $2.74 \mathrm{~Hz}$, where the amplitude was $10 \mathrm{~mm}$. 
During the laboratory tests, the tip of the beam displacement was measured by the accelerometer. Typical experimental results are depicted in Fig. 4. Captured and simmulated waveforms of the investigated element (Figs. 4a and 4b) correspond to various actiavation times: $4.5 \mathrm{~s}$ and $1.5 \mathrm{~s}$, respectively. In this preliminary approach, the mass of granular material $M_{p}$ was constant and assumed as $10 \%$ of the global system.

Basing on the verification of experimental and numerical data, it can be found that the activation of ATPID elements reasonably reduces the resonance amplitudes. We can also observe that the real behavior of the beam slightly differs from the numerically calculated response. Although the previously mentioned assumption related to the linear character of $C_{A T P I D}$ parameter changes in function of the compartment volume is valid for the "turning on" state, the real behavior of the system is more complex than the model response. It is specially visible in the "turning off" state, where nonlinearity of the "viscous" phenomenon is observable.

\section{Conslusions}

This paper presents an innovative Particle Impact Damper design that thanks to the possibility of controlling in real time the volume of the device allows for introducing new semi-active damping strategies. The detailed construction and laboratory tests of the ATPID damper was presented and its working rule was discussed.

In the activation function (Fig. 3), the turning "on" and "off" time was assumed as 4.5 and 1.5 seconds. The experimentally measured time was $5.4 \mathrm{~s}$ and $1.9 \mathrm{~s}$, respectively. The activation stage for the ATPID is well represented by the simplest 2-DOF mathematical model. More visible discrepancies are observed in the deactivation process where "inertia" of the granular media and the inelastic character of intergranular collisions play a more important role. It is worth mentioning that the application of the ATPID element reduced the resonance amplitude of the system by more than $40 \%$.

The proposed innovative damping strategy can be easily implemented in various dynamical systems consisting of long, slender and light elements such as wind turbines or other civil engineering applications. ATPID devices seem to be especially efficient in damping of resonant frequencies of various shaped elements working in demanding environmental conditions such as high temperatures.

\section{References}

1. Bartkowski P., Zalewski R., Chodkiewicz P., 2019, Parameter identification of Bouc-Wen model for vacuum packed particles based on genetic algorithm, Archives Of Civil And Mechanical Engineering, 19, 322-333

2. Chen J., Georgakis C.T., 2013, Tuned rolling-ball dampers for vibration control in wind turbines, Journal of Sound and Vibration, 332, 5271-5282

3. DU Y., 2017, A spring-supported fine particle impact damper to reduce harmonic vibration of cantilever beam, Advances in Mechanical Engineering, 9, 5

4. Du Y., WAng S., 2010, Modeling the fine particle impact damper, International Journal of Mechanical Sciences, 52, 1015-1022

5. Fitzgerald B., Sarkar S., Staino A., 2018, Improved reliability of wind turbine towers with active tuned mass dampers (ATMDs), Journal of Sound and Vibration, 419, 103-122

6. Hussan M., Rahman M.S., Sharmin F., Kim D., Do J., 2018, Multiple tuned mass damper for multi-mode vibration reduction of offshore wind turbine under seismic excitation, Ocean Engineering, 160, 449-460 
7. Lei X., Wu C., Chen P., 2018, Optimizing parameter of particle damping based on Leidenfrost effect of particle flows, Mechanical Systems and Signal Processing, 104, 60-71

8. Paget A.L., 1937, Vibration in steam turbine buckets and damping by impacts, Engineering, 143, 305-307

9. Snoun C., Trigui M., 2018, Design parameters optimization of a particles impact damper, International Journal on Interactive Design and Manufacturing (IJIDeM), 12, 1283-1297

10. Zalewski R., Żurawski M., Chodkiewicz P., 2019, Passive-adaptive vibration damper with loose grain material (in Polish), Patent P.430075

11. Zhang K., Chen T., Wang X., Fang J., 2016, Rheology behavior and optimal damping effect of granular particles in a non-obstructive particle damper, Journal of Sound and Vibration, 364, $30-43$

12. Zhang Z., Staino A., Basu B., Nielsen S.R., 2016, Performance evaluation of full-scale tuned liquid dampers (TLDs) for vibration control of large wind turbines using real-time hybrid testing, Engineering Structures, 126, 417-431

Manuscript received August 26, 2019; accepted for print January 7, 2020 